\title{
Random Lasing at Localization Transition in a Colloidal Suspension ( $\mathrm{TiO}_{2} @$ Silica)
}

\author{
Ernesto Jiménez-Villar, ${ }^{*}+\oplus$ Iran F. da Silva, ${ }^{\dagger}$ Valdeci Mestre, ${ }^{\|}$Niklaus U. Wetter, ${ }^{\perp}$ Cefe Lopez, \\ Paulo C. de Oliveira, ${ }^{\$ ®}$ Wagner M. Faustino, ${ }^{\ddagger}$ and Gilberto F. de Sá ${ }^{\dagger}$
}
${ }^{\dagger}$ Departamento de Química Fundamental, Universidade Federal de Pernambuco, Cidade Universitária, 50670-901 Recife, PE, Brazil ${ }^{\ddagger}$ Departamento de Química and ${ }^{\S}$ Departamento de Física, Universidade Federal da Paraíba, Cidade Universitária, 58051-970 João Pessoa, PB, Brazil
"Universidade Estadual da Paraíba, CCEA, Rua Alfredo Lustosa Cabral, s/n, 58706-560 Patos, PB, Brazil
${ }^{\perp}$ Instituto de Pesquisas Energéticas e Nucleares, CNEN-IPEN, Rua Prof. Lineu Prestes 2242 - Cidade Universitária, 05508-000 São Paulo, SP, Brazil
${ }^{\#}$ Instituto de Ciencia de Materiales de Madrid (CSIC), Sor Juana Inés de la Cruz, 3, 28049 Madrid, Spain

\section{Supporting Information}

\begin{abstract}
Anderson localization of light and random lasing in this critical regime is an open research frontier, which besides being a basic research topic could also lead to important applications. This article investigates the random laser action at the localization transition in a strongly disordered scattering medium composed of a colloidal suspension of core-shell nanoparticles $\left(\mathrm{TiO}_{2} @\right.$ Silica $)$ in ethanol solution of Rhodamine 6G. The classical superfluorescence band of the random laser was measured separately by collecting the emission at the back of the samples, showing a linear dependence with pumping fluence without gain depletion. However, frontal collection showed saturation of the absorption and emission. Narrow peaks of

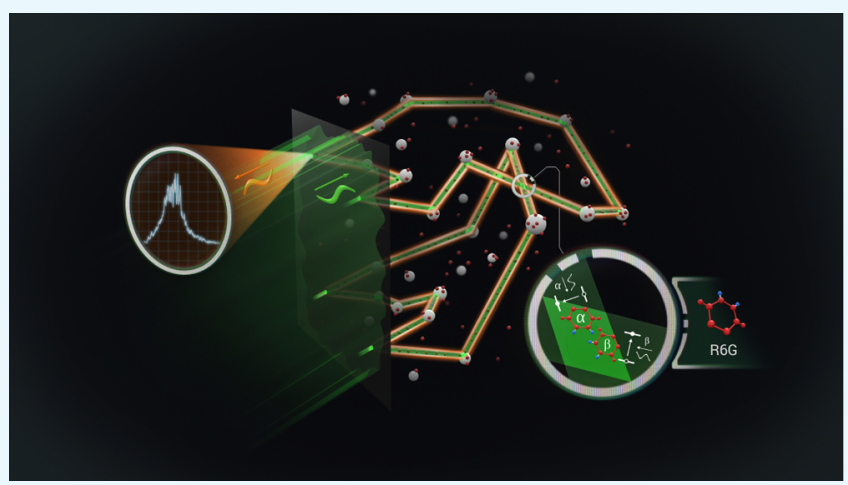
approximately equal intensity are observed on top of the classical superfluorescence band, indicating suppression of the interaction between the peaks modes. The linewidth of these peaks is lower than that of the passive modes of the scattering medium. A method called fraction of absorbed pumping allowed us to infer that this peak's mode (localized modes) is confined to a shallow region near the input-pumping border.
\end{abstract}

\section{INTRODUCTION}

Scattering media have attracted much attention in recent years, ranging from investigations into fundamental topics, such as light confinement ${ }^{1-5}$ and other phenomena involving photon interactions, ${ }^{6,7}$ to their potential applications in solar energy, ${ }^{8}$ photocatalyzers, ${ }^{9}$ and random lasers (RL). ${ }^{10-12}$ Localization of light and a wide variety of associated phenomena have greatly attracted the attention of researchers in the past decades. In a previous work, ${ }^{5}$ we reported several pieces of experimental evidence of localization transition in a colloidal suspension composed of $\mathrm{TiO}_{2} @$ Silica nanoparticles (NPs) in ethanol solution. By using the Stöber method, ${ }^{13,14} \mathrm{TiO}_{2}$ NPs were coated with a homogeneous silica shell of $\sim 40 \mathrm{~nm}$ thickness. The silica shell provides optical colloidal stability (OCS), ${ }^{15,16}$ light-coupling enhancement with the $\mathrm{TiO}_{2}$ scatterer core, ${ }^{17}$ high dispersibility of NPs, ${ }^{18-20}$ and inertness, ${ }^{21,22}$ which have enabled their use in numerous applications. ${ }^{23-25}$ The silica coating with thicknesses above $\sim 40 \mathrm{~nm}$ prevents the "optical" junction of the $\mathrm{TiO}_{2}$ scattering surfaces (steric "optical" effect), ${ }^{15}$ decreasing considerably the near-field coupling, which decreases the scattering strength and could hamper localization. ${ }^{16}$ We called this property OCS. ${ }^{15}$ The core-shell $\mathrm{TiO}_{2} @$ Silica NPs allowed us to obtain a liquid suspension with a significantly higher scattering strength than pure rutile particles. Transport experiments in this $\mathrm{TiO}_{2} @$ Silica colloidal suspension showed a crossover from diffusive light propagation to localization transition as the NP concentration increased, and an enhanced absorption at the localization transition was observed. ${ }^{5}$ The study of the RL action at this critical regime of approaching localization is an open research frontier. It has been shown that a scattering medium with gain at the localization or localization transition can lead to random lasing with coherent feedback, ${ }^{26,27}$ which besides being a fundamental topic $^{11}$ could also present significant applications. ${ }^{28}$ Another alternative scattering medium for localization of light and random nanolasing (spaser) has been the plasmonic NPs,

Received: January 23, 2017

Accepted: March 20, 2017

Published: June 1, 2017 

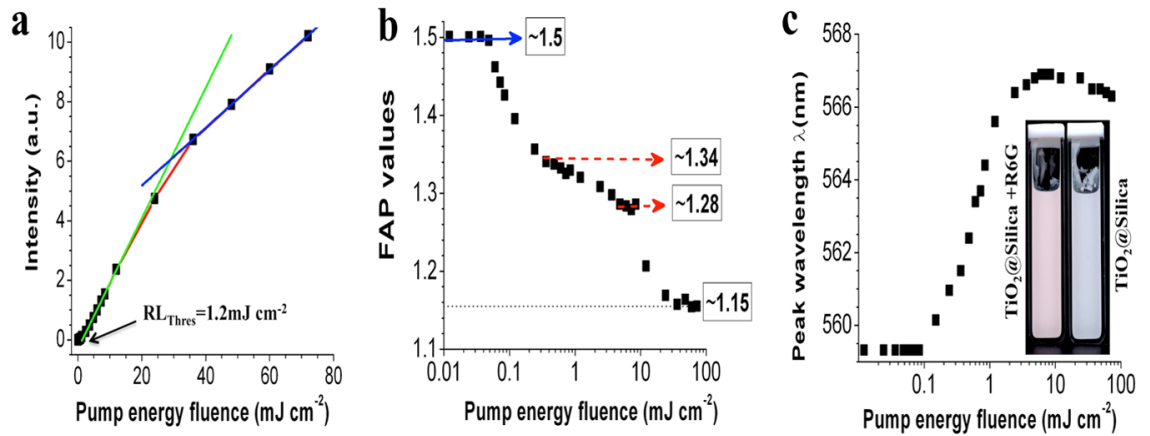

Figure 1. Influence of the pumping fluence on (a) the $\mathrm{RL}$ emitted peak intensity, $\mathrm{RL}_{\text {eff, }}$ for fluencies $<12 \mathrm{~mJ} \mathrm{~cm}{ }^{-2}$ (green line) and $>36 \mathrm{~mJ} \mathrm{~cm}^{-2}$ (blue line), (b) the $\mathrm{FAP}_{\mathrm{bT}}$ (blue arrow) and (c) the peak position of the emission spectrum; inset: pictures of the $\mathrm{TiO}_{2} @ S$ Silica suspensions with and without gain (R6G).

which was experimentally demonstrated by Noginov and coworkers. $^{29}$

In this work, random lasing is studied at the localization transition. Narrow peaks arising in the RL emission spectrum are observed. This kind of peaks has been reported in systems composed by solid powders, ${ }^{30,31}$ porous gallium phosphide filled with liquid dye solution, ${ }^{32}$ sparse clusters, ${ }^{33}$ photonic crystals, ${ }^{34,12}$ and in low dimensional systems. ${ }^{35-37}$ Spikes have also been observed over a broad range of scattering strengths. ${ }^{38}$ However, recent works ${ }^{39,40}$ claim that, depending on the relative magnitude of the localization length and the system size, quasi modes and lasing modes may not be the same, and the statistical distribution of these emission spikes is an indication that under certain pumping conditions they do not correspond to true lasing with coherent feedback. Detailed reviews on this topic have been published elsewhere. ${ }^{41,42}$

\section{RESULTS AND DISCUSSION}

Analysis of Laser Emission. Figure 1a shows the behavior of the emitted intensity as a function of pumping energy fluence. Typical RL emission spectra and the behavior of the spectral width (full width at half-maximum (FWHM)) can be found in Figure S2. From Figure 1a, we can observe that the RL slope efficiency $\left(\mathrm{RL}_{\text {eff }}\right)$ is not constant; it decreases markedly for pumping fluencies from $\sim 12$ up to $36 \mathrm{~mJ} \mathrm{~cm}{ }^{-2}$, showing a saturation of the emission. The green line represents the $\mathrm{RL}_{\text {eff }}$ for fluencies $<12 \mathrm{~mJ} \mathrm{~cm}^{-2}$. For pumping fluencies $\geq 36 \mathrm{~mJ}$ $\mathrm{cm}^{-2}, \mathrm{RL}_{\text {eff }}$ adopts a 2.3 times smaller value (blue line). The $\mathrm{RL}$ threshold, determined by extrapolating the straight line corresponding to $\mathrm{RL}_{\text {eff }}$ (green line) to a zero emission intensity, is $\sim 1.2 \mathrm{~mJ} \mathrm{~cm}^{-2}$ (Supporting Information). The light of the pumping pulse reflected by the samples was measured with and without R6G. We designated the ratio between the pumping intensities reflected by the scattering medium with and without R6G as the fraction of absorbed pumping (FAP), which represents the absorbance of the sample, $\ln (\mathrm{FAP}) .{ }^{15} \mathrm{FAP}$ dependence with pumping energy fluence is shown in Figure $1 \mathrm{~b}$. The FAP value for fluencies $<0.048 \mathrm{~mJ} \mathrm{~cm}^{-2}$, well below the laser threshold $\left(\mathrm{FAP}_{\mathrm{bT}}\right)$, was constant $(\sim 1.5)$. The FAP value decreases quickly for fluencies of 0.048 up to $0.36 \mathrm{~mJ} \mathrm{~cm}^{-2}$. For fluencies between 0.36 and $4.8 \mathrm{~mJ} \mathrm{~cm}^{-2}$, the FAP values decrease slowly from 1.34 down to 1.28 , where it remains approximately constant up to a fluence of $8.4 \mathrm{~mJ} \mathrm{~cm}^{-2}$. The FAP values start to drop quickly again for fluencies $>8.4 \mathrm{~mJ}$ $\mathrm{cm}^{-2}$ and decrease down to $\sim 1.15$ for fluencies $\geq 36 \mathrm{~mJ} \mathrm{~cm}^{-2}$, showing a clear phase transition for the absorption, indicating two different modes of laser operation. From the $\mathrm{FAP}_{\mathrm{bT}}$ value
(1.5), we can estimate the average photon path length $\left(l_{\mathrm{eO}}\right)$ inside the scattering medium before being reflected, as well as the photon residence time $\left(\tau_{\mathrm{eO}}\right) .{ }^{15}$

$$
\begin{aligned}
l_{\mathrm{eO}} & =\frac{l_{\mathrm{a}}}{\left(\frac{1+\gamma_{0}}{2}\right)} \ln \left(\mathrm{FAP}_{\mathrm{bT}}\right) \\
\tau_{\mathrm{eO}} & =\frac{l_{\mathrm{eO}}}{c} n_{\mathrm{effo}} \\
& =l_{\mathrm{a}} \frac{\ln \left(\mathrm{FAP}_{\mathrm{bT}}\right)}{\left(\frac{1+\gamma_{0}}{2}\right) c} n_{\mathrm{eff} 0} \\
& =l_{\mathrm{a}} \frac{\ln \left(\mathrm{FAP}_{\mathrm{bT}}\right)}{\left(\frac{1+\gamma_{0}}{2}\right) c}\left(1+\left(\frac{1+\gamma_{0}}{2}\right)\left(n_{\mathrm{eff}}-1\right)\right)
\end{aligned}
$$

where $l_{a}, \gamma_{0}, n_{\text {effo, }} n_{\text {eff }}$ and $c$ are the ballistic or microscopic absorption length [R6G], the enhanced absorption factor by localization $\left(\gamma_{0}=1.84\right)$, the effective refractive index (increased by localization), the classical refractive index (1.53), and speed of light, respectively. The $l_{\mathrm{a}}$ value well below the laser threshold (passive regime) should not change $\left(384 \mu \mathrm{m}\right.$ at $\left[10^{-4} \mathrm{M}\right]$ of $\left.\mathrm{R} 6 \mathrm{G}\right)$. However, just as it was determined in our previous work, ${ }^{5}$ the absorption coefficient and the effective refractive index are enhanced near the inputpumping surface $n_{\text {effo }}=1+\left(\frac{1+\gamma_{0}}{2}\right)\left(n_{\text {eff }}-1\right)$. The term $\left(\frac{1+\gamma_{0}}{2}=\frac{1+1.84}{2}=1.42\right)$ represents an estimate of the mean value of the enhanced absorption factor in the region where absorption is enhanced (Supporting Information). In this way, the photon path length and the photon residence time are

$$
l_{\mathrm{eO}}=\frac{384 \mu \mathrm{m}}{1.42} \times \ln (1.5) \approx 110 \mu \mathrm{m}
$$

and

$$
\begin{aligned}
\tau_{\mathrm{eO}} & =\frac{384}{1.42} \times 3.3 \times 10^{-3} \mathrm{ps} \times 0.41 \times(1+1.42 \times(1.53-1)) \\
& \approx 0.64 \mathrm{ps}
\end{aligned}
$$

respectively. In this calculus, we have not considered that a portion of the light, although to a lesser extent, could also come from a region deeper inside the sample. Notice that this experimental setup collects the light coming from the whole pumped volume $(\sim 15 \mu \mathrm{m}$ in depth, see Supporting Information). We note that $l_{\mathrm{eO}}$ is much lower than the 

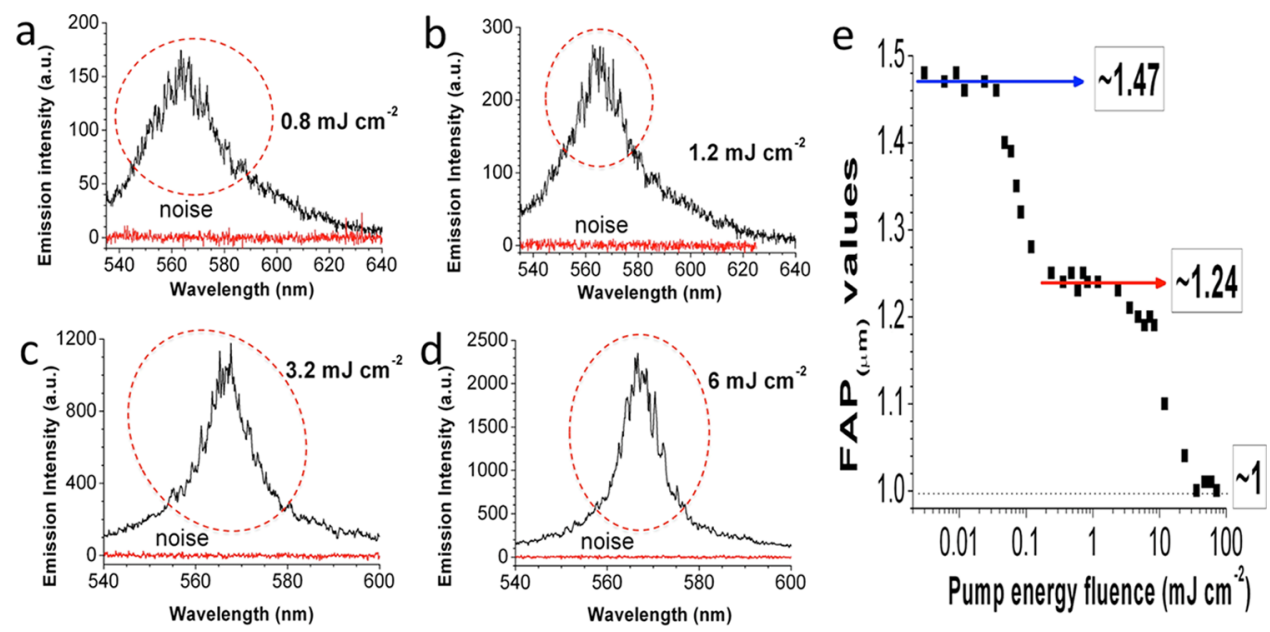

Figure 2. Emission spectra of RL collected from a small micrometric volume ( $<4 \mu \mathrm{m}$ diameter and depth) for pumping fluencies of (a) 0.8 , (b) 1.2 , (c) 3.2 , and (d) $6 \mathrm{~mJ} \mathrm{~cm}^{-2}$. The diameter of the pumping spot is the same $(3 \mathrm{~mm})$. The solid red lines represent the respective noise signal. The dashed red circles highlight peaks emerging in the RL emission spectrum. Emission spectra were collected integrating 21 laser shots. (e) FAP from the micrometric volume $\left(\operatorname{FAP}_{(\mu \mathrm{m})}\right)$, as a function of the pumping energy fluence. $\operatorname{FAP}_{(\mu \mathrm{m})} \approx 1$ for pumping fluencies $\geq 36 \mathrm{~mJ} \mathrm{~cm}^{-2}$.

microscopic absorption length of the scattering medium near the sample's surface $\left(l_{\text {In0 }}\right)$ (residual “absorption” without R6G) determined in our previous work $\left(l_{\mathrm{In} 0} \approx 2700 \mu \mathrm{m} \gg l_{\mathrm{eO}} \approx 110\right.$ $\mu \mathrm{m}){ }^{5}$ Therefore, the photons would leave the scattering medium long before being absorbed by it.

The RL emission peak (Figure 1c) shows a redshift that increases rapidly for fluencies from 0.084 up to $8.4 \mathrm{~mJ} \mathrm{~cm}^{-2}$. For fluencies between 8.4 and $24 \mathrm{~mJ} \mathrm{~cm}^{-2}$, the redshift is approximately constant, which coincides with the starting decrease of $\mathrm{RL}_{\text {eff }}$ (Figure 1a) and FAP or RL absorbance $\left(\mathrm{RL}_{\mathrm{Abs}}\right)$ (Figure $1 \mathrm{~b}$ ). The redshift starts to decrease for fluencies $\geq 36 \mathrm{~mJ} \mathrm{~cm}^{-2}$, for which the $\mathrm{RL}_{\text {eff }}$ and $R L_{\text {Abs }}$ reach the minimum value. The maximum redshift value $(\sim 7.6 \mathrm{~nm})$ is large, especially considering that the absorption and emission are both saturated, when compared with the customary maximum redshift for a $\mathrm{TiO}_{2} \mathrm{NP}$ system, which is around 3$4 \mathrm{~nm}$. For comparison, the $\mathrm{TiO}_{2} @$ Silica system at low [NPs] (diffusive regime) showed a maximum redshift of less than 1 nm. ${ }^{15,17}$ The redshift was previously explained by a model considering absorption and emission at the transition between the ground state and the first excited singlet state of the dye molecule: ${ }^{43}$

The observed saturated absorption (Figure 1b) can be explained in the following way: a very low percentage of R6G molecules are in the lowest sublevels, $S_{0 i}$, of the ground state, and/or a high population of molecules are in the sublevels, $S_{1 j}$ of the excited singlet state. Saturated emission (Figure 1a) means a low population of molecules are in the lowest sublevels, $S_{1 i}$, of the excited singlet state or/and a high population are in the $S_{0 i}$ sublevels of the ground state. Joining saturated absorption and saturated emission, the result is that a large percentage of R6G molecules are encountered in the higher lying sublevels of $S_{1}$ and $S_{0}$. Therefore, the transition rates from $S_{0 \mathrm{i}}$ up to $S_{1 \mathrm{i}}$ (absorption) and from $S_{1 \mathrm{i}}$ to $S_{0 \mathrm{i}}$ (stimulated emission) are higher than the vibrational relaxation rate within the excited singlet sublevels, $S_{1 j}$ or within the sublevels of the ground state, $\mathrm{S}_{0 \mathrm{i}}$ (see Supporting Information). The polarization of $\mathrm{R} 6 \mathrm{G}$ molecules by pumping photons trapped within the closed loop paths (localized state) should give rise to a photon-molecule bound state, ${ }^{44,45}$ which in turn would lead to the suppression of vibrational relaxation and spontaneous emission. ${ }^{46}$

RL Action in a Small Micrometric Volume. When collecting the light from the front side of the sample coming from a large surface area as in Chapter 3, the emission of localized modes is integrated, making its individual detection difficult. Therefore, to better analyze the possible peaks, a study has been performed collecting $\mathrm{RL}$ emissions from a micrometric region $(\sim 3.8 \mu \mathrm{m}$ diameter $)$ near the input-pumping surface (less than $4 \mu \mathrm{m}$ depth) using a detection system that performs like a confocal microscope. For the experimental setup, see Figure S1b. Figure 2 shows the evolution of the emission spectra for pumping fluencies below $0.8 \mathrm{~mJ} \mathrm{~cm}$, close to $1.2 \mathrm{~mJ} \mathrm{~cm}^{-2}$, and above the RL threshold, 3.2 and $6 \mathrm{~mJ}$ $\mathrm{cm}^{-2}$. The spectral region framed with a dashed circle highlights the emerging peaks. For lower pumping fluencies $(0.8 \mathrm{~mJ}$ $\mathrm{cm}^{-2}$ ), the spectra consisted of the fluorescence emission band with overlapping narrow peaks at frequencies close to the maximum of the spectrum. As the pump fluence increases, the emission band becomes narrower, although the overlapped narrow peaks continue to appear in approximately the same frequency interval of $15-20 \mathrm{~nm}$. The minimum linewidth observed for the narrowest peaks was $\leq 0.17 \mathrm{~nm}$, which is the spectral resolution limit of our detection system. The position of these sharp peaks changes little in a few seconds; it only changes completely after tens of seconds or by stirring the sample. Notice that the pump spot is $3 \mathrm{~mm}$ in diameter, and the estimated pump depth above the RL threshold must be around $15 \mu \mathrm{m}$ (Supporting Information). Therefore, the effects caused by inhomogeneous pumping should not play a role in promoting these peaks. In addition, the narrow peaks could not result from amplified spontaneous emission, ${ }^{38}$ as these emission spectra are obtained by integrating 21 laser shots of long duration $(\sim 4 \mathrm{~ns})$. We must highlight that the intensity of these narrow peaks, relative to the fluorescence band, is lower than that reported by Cao and co-workers; ${ }^{27}$ however, similar peaks have been observed by Gagné and Kashyap ${ }^{36}$ and Cao and coworkers in a subsequent work. ${ }^{31}$ An expanded discussion about these peaks, its intensity, and density can be found in the Supporting Information. 

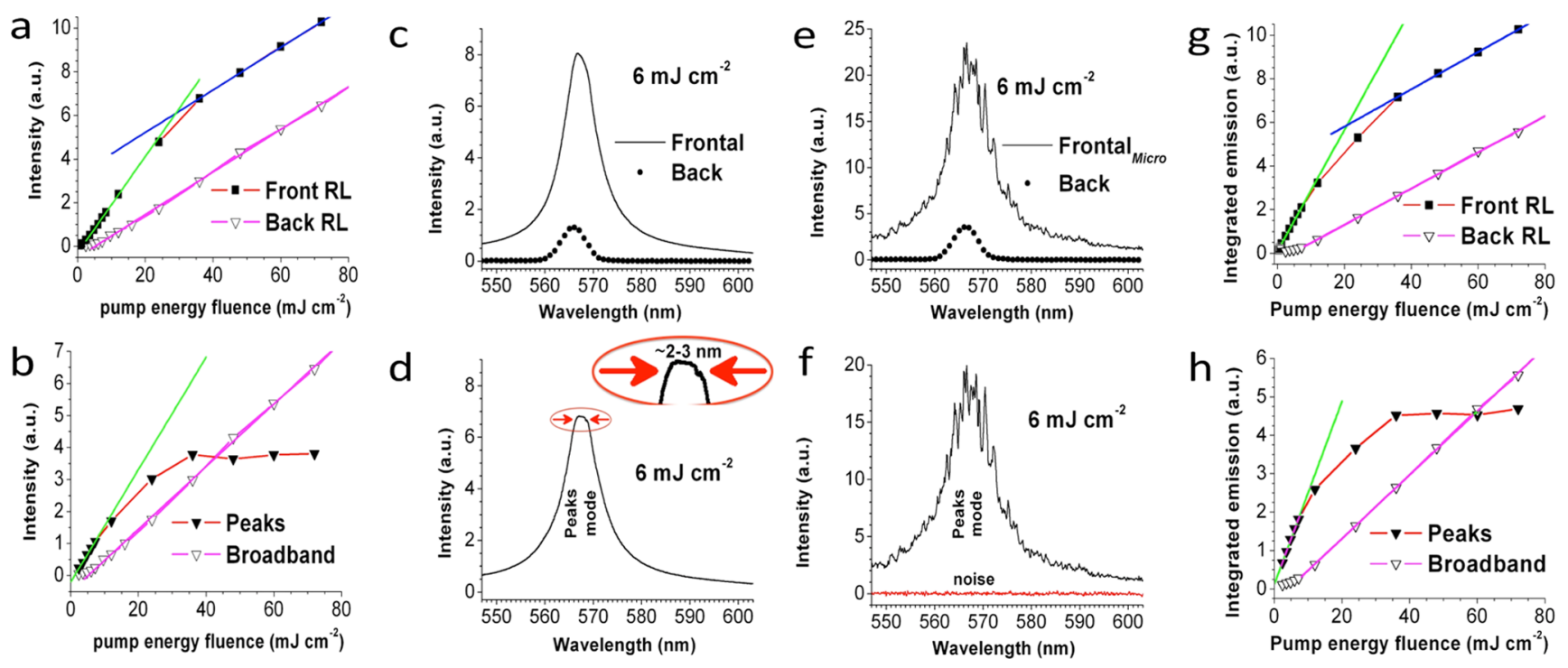

Figure 3. Influence of the pump fluence on (a) emitted peak intensity for frontal and back collection, (b) emitted peak intensity of RL back collection (open triangle) and difference between frontal and back collection (closed triangle). (c) The emission spectra for a fluence of $6 \mathrm{~mJ} \mathrm{~cm}^{-2}$ collected at the back (dots) and front (line) of the cuvette, and (d) difference between the frontal and back spectra of (c). (e) The emission spectra for fluence of $6 \mathrm{~mJ} \mathrm{~cm}{ }^{-2}$ recorded by back collection (dots) and the frontal collection in a small micrometric volume (Figure 2d), and (f) difference between both spectra. (g) and (h) are similar to (a) and (b), respectively, but are the spectrally integrated emission intensity.

The FAP values from the small micrometric volume $\left(\mathrm{FAP}_{(\mu \mathrm{m})}\right)$ were measured with the same experimental setup (Figure $\mathrm{S} 1 \mathrm{~b})$. Figure 2e shows the $\mathrm{FAP}_{(\mu \mathrm{m})}$ dependence with the pumping fluence. The $\operatorname{FAP}_{(\mu \mathrm{m})}$ values for fluencies $(<0.036$ $\mathrm{mJ} \mathrm{cm} \mathrm{cm}^{-2}$ ) well below the laser threshold $\left(\mathrm{FAP}_{\mathrm{bT}(\mu \mathrm{m})}\right)$ are constant $(\sim 1.47)$, which represents a passive regime. For fluencies between 0.036 and $0.24 \mathrm{~mJ} \mathrm{~cm}$, the $\mathrm{FAP}_{(\mu \mathrm{m})}$ value decreases quickly down to $\sim 1.24$, where it remains approximately constant up to a fluence of $\sim 2.4 \mathrm{~mJ} \mathrm{~cm}^{-2}$. The latter implies that for this range of fluencies $(0.24-2.4 \mathrm{~mJ}$ $\mathrm{cm}^{-2}$ ), a stationary regime is reached in this micrometric volume near the input-pumping surface ( $<4 \mu \mathrm{m}$ depth). The $\operatorname{FAP}_{(\mu \mathrm{m})}$ value starts to drop again quickly for fluencies $>8.6 \mathrm{~mJ}$ $\mathrm{cm}^{-2}$ and decrease down to $\sim 1$ (null absorbance) for fluencies $\geq 36 \mathrm{~mJ} \mathrm{~cm}{ }^{-2}$. This means that absorbance in this micrometric volume (near the input-pumping surface) becomes practically zero for fluencies $\geq 36 \mathrm{~mJ} \mathrm{~cm}^{-2}$. However, the photon residence time in this micrometric volume $\left(\tau_{\mathrm{eO} \text { (Micro) }} \approx 0.612 \mathrm{ps}\right.$; eq 2$)$ would yield a homogeneous spectral broadening of $\sim 1.29 \mathrm{~nm}$, obtained by using a Fourier transform assuming a Gaussian envelope, which is broader than the narrowest linewidth observed in the RL peaks $(\leq 0.17 \mathrm{~nm})$. This fact could be a consequence of an anomalous nonlinear increase of the refractive index within the closed loop paths (localized states), which gives rise to a phase shift (for the pumping photon) that continuously increases by increasing the intensity (within the closed loop paths), during the residence time of the pumping photons. This phase accumulated can lead to a resonance breaking, reducing $\tau_{\mathrm{eO} \text { (Micro) }}$. This phase accumulation by such an anomalous nonlinear effect would be greatly suppressed for emitted photons above the lasing threshold, as the intensity increase for each lasing mode would be a consequence of the stimulated emission. In other words, the phase accumulated within the localized state during the laser action (emission) by nonlinear effects is corrected continually, as the intensity increase results from the stimulated emission. Perhaps for this reason, the coherent laser emission sustained by localization has been largely recognized in disordered optical media with gain. $^{27,29-31,34}$ However, the direct observation of localization in the absence of gain has proved elusive. ${ }^{3,4}$ In this way, an anomalous nonlinear increase of the refractive index could be a likely cause, by which pump photons leave the scattering medium, breaking the interference condition (localization). A similar nonlinear phenomenon was theoretically addressed by Buttiker and Moskalets in disordered electronic media, ${ }^{47}$ who proposed that when the energy of the localized state changes, the localized state can emit nonequilibrium electrons and holes propagating away from the localized state within the edge state, which acts similar to a waveguide.

RL Mode Study. To determine the contribution of the RL modes, the superfluorescence band (extended band mode or ASE), and the narrow peaks (peaks mode), an additional experiment was designed. Let us introduce the following conjecture: if the narrow peaks are derived from localized modes due to the localization phenomenon, then they should basically be backscattered, that is, their emission should be detected in the direction opposite to the pump direction. Note that the emission of localized modes inside the sample is exponentially attenuated, so that if they are very near the front edge, they will be detected only in the spectrum obtained from the front surface (frontal spectrum) because they are too far from the back edge to survive, as the attenuation is huge.

During the experiment, the RL emission was also collected from the back of the cuvette (back collection). The backcollected spectra consisted of a broad emission band $(\sim 50 \mathrm{~nm}$ bandwidth) with an overlapped single emission band (dotted trace in Figure 3c,e), whose intensity and bandwidth are dependent on the pumping energy (Figure S5). Figure 3a shows the behavior of the RL intensity as a function of the pumping fluencies for back and frontal collection. The RL slope efficiency $\left(\mathrm{RL}_{\text {eff }}\right)$ for back collection $\left(\mathrm{RL}_{\text {eff-back }}\right)$ was constant without depletion (magenta line), whereas the $\mathrm{RL}_{\text {eff }}$ of frontal collection decreases from fluencies $\geq 12$ up to $36 \mathrm{~mJ} \mathrm{~cm} \mathrm{~cm}^{-2}$, where it keeps constant (blue line, fluencies $\geq 36 \mathrm{~mJ} \mathrm{~cm}^{-2}$ ).

Owing to the RL peaks mode being saturated at fluencies $\geq 36 \mathrm{~mJ} \mathrm{~cm}^{-2}$, we may consider that the saturated $\mathrm{RL}_{\text {eff }}$ of the 
frontal collection (blue line, Figure 3a) corresponds to the $\mathrm{RL}_{\text {eff }}$ of the extended band mode ( $\left.\mathrm{RL}_{\text {eff- } \mathrm{B}}, \mathrm{ASE}\right)$. Therefore, we rescaled the experimental points of the back collection, which is also ASE or the band mode, by a mathematical factor, such that $R L_{\text {eff-back }}=R L_{\text {eff- } \mathrm{B}}$. The $R L$ emission intensity corresponding to the peaks mode (localized modes) can then be determined from the difference in intensities between frontal and back collection. Figure $3 \mathrm{~b}$ shows a comparison of the RL intensity as a function of the pumping fluence for the peaks mode (frontalback) and back-collected mode. The $\mathrm{RL}_{\text {eff }}$ peaks mode $\left(\mathrm{RL}_{\text {eff-P }}\right)$ for fluencies $<12 \mathrm{~mJ} \mathrm{~cm}^{-2}$ (green line) was 1.8-fold higher than the $\mathrm{RL}_{\text {eff-back, }}$ and $R L_{\text {eff-P }} \approx 0$ for fluencies $\geq 36 \mathrm{~mJ} \mathrm{~cm}^{-2}$. Thus, the contribution of the peaks mode as compared to the band mode decreases remarkably as the fluence is increased above 36

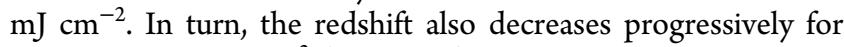
fluencies $\geq 36 \mathrm{~mJ} \mathrm{~cm}^{-2}$ (Figure 1c). Therefore, we associate the large redshift $(\sim 7.6 \mathrm{~nm})$ observed in the $\mathrm{RL}$ emission to the emission of the peaks mode (localized modes). The RL threshold for the band mode, determined from extrapolating $\mathrm{RL}_{\text {eff- } \mathrm{B}}$ to zero, is $\sim 4 \mathrm{~mJ} \mathrm{~cm}^{-2}$. The latter explains the slow FAP decrease observed in Figure $1 \mathrm{~b}$ for fluencies between 0.36 and $4.8 \mathrm{~mJ} \mathrm{~cm}^{-2}$. Moreover, this implies that the RL threshold of the peaks mode must be well below that of the band mode. Notice that the RL threshold from frontal collection (peaks + band) is only $1.2 \mathrm{~mJ} \mathrm{~cm}^{-2}$.

Figure $3 \mathrm{c}$ shows the $\mathrm{RL}$ emission spectra at $6 \mathrm{~mJ} \mathrm{~cm}^{-2}$ for both collections, the frontal spectrum collected over the whole emission volume and the back spectrum. The bandwidth of the frontal spectrum $(8.8 \mathrm{~nm})$ is larger than that of the back spectrum $(4.8 \mathrm{~nm})$. In addition, the frontal spectra are slightly asymmetrical, showing a widening toward a longer wavelength. The resulting spectrum from the difference between both spectra (Figure 3d), which must correspond to the emission spectrum of the peaks mode collected from the whole emission area (integrated collection), shows a plateau in intensity of 2-3 $\mathrm{nm}$ (for other pumping fluencies, see Figure S6). This indicates suppression of the interaction between the peaks modes, which is a signature of random lasing at localization. ${ }^{11}$ Figure S7 shows a representative picture of this phenomenon of suppression of the interaction between localized modes.

For fluencies $\geq 36 \mathrm{~mJ} \mathrm{~cm}^{-2}$, both $\mathrm{RL}_{\text {eff-P }}$ and the absorbance in the small micrometric volume near the input-pumping surface $\left(\ln \left(\mathrm{FAP}_{(\mu \mathrm{m})}\right)\right)$ are approximately zero $\left(\mathrm{RL}_{\text {eff-P }}=0\right.$ and $\left.\operatorname{FAP}_{(\mu \mathrm{m})} \approx 1\right)$, corresponding to absorption and emission saturation. Therefore, it can be inferred that pumping and emission of the peaks mode occurs mainly in this micrometric volume near the input-pumping border. However, FAP (integrated collection) decays down to 1.15 for pumping fluencies $\geq 36 \mathrm{~mJ} \mathrm{~cm}{ }^{-2}$ (Figure 1c), where it remains approximately constant. This means that a stationary lasing regime is reached for a depth $>4 \mu \mathrm{m}$ and for pumping fluencies $\geq 36 \mathrm{~mJ} \mathrm{~cm}^{-2}$. From the latter it can be interpreted that, near the input-pumping surface $(<4 \mu \mathrm{m}$ depth), the absorptionemission contribution of the nonlocalized modes must be negligible as compared to that of the peaks mode (localization mode), that is, the nonlocalized modes should mainly be located deeper in the sample $(>4 \mu \mathrm{m})$.

The R6G microscopic absorption length (small micrometric volume near the input-pumping border) above the RL threshold $\left(l_{\mathrm{a}^{*}(\mu \mathrm{m})}\right)$ is $\sim 483 \mu \mathrm{m}$ (see Supporting Information). Furthermore, the average photon path length, $l_{\mathrm{eO}}$, in this micrometric volume near the input-pumping border $\left(l_{\mathrm{eO}(\mathrm{Micro})}\right)$ is $\sim 104 \mu \mathrm{m}$ (eq 1$)$. Therefore, $l_{\mathrm{eO}(\text { Micro })}<l_{\mathrm{a} *(\mu \mathrm{m})}$, which means that above the $\mathrm{RL}$ threshold the pumping photons leave the scattering medium (backscattered) long before being absorbed by $\mathrm{R} 6 \mathrm{G}$ molecules. As a consequence, the R6G absorption

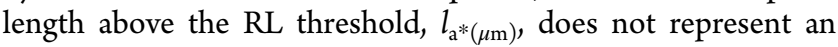
impediment for localizing pumping photons. The latter indicates that the frequency and wavefunction of a localized lasing mode should be approximately the same as those of the passive mode of the scattering medium, which was theoretically predicted by Stano and Jacquod. ${ }^{11}$

Figure 3e shows the RL emission spectra at $6 \mathrm{~mJ} \mathrm{~cm}{ }^{-2}$ for frontal collection (from a small micrometric volume near the input border) and back collection. The bandwidth of the frontal spectrum $(\sim 9.6 \mathrm{~nm})$ is larger than the back spectrum $(4.8 \mathrm{~nm})$. Figure $3 \mathrm{f}$ shows the spectrum resulting from the difference between both spectra, which corresponds to the emission spectrum of the peaks mode coming from a small micrometric volume near the input-pumping border $(<4 \mu \mathrm{m}$ depth). The narrow peaks in Figure $3 \mathrm{f}$ are more defined, showing similar intensities in a large frequency range $(\sim 7-8 \mathrm{~nm})$, presumably due to the lower coupling degree and higher gain of the peaks mode, ${ }^{11}$ allowing for the lasing condition in a large frequency range within the R6G fluorescence bandwidth. The similarity in intensity of the narrow peaks could be caused by (i) the stimulated emission rate being higher than the vibrational relaxation rate, causing the gain of the peaks to be approximately equal in this frequency range, (ii) suppression of the interaction between the peaks modes. Notice that, owing to localization, spontaneous emission and vibrational relaxation must be inhibited (within localized states), ${ }^{44-46}$ and the interaction between localized lasing modes (peaks mode) would be considerably suppressed, even between modes that have significant spatial or frequency overlap. ${ }^{11,12}$ The latter can be understood by realizing that the photons confined in a localized mode must be strongly correlated. ${ }^{11}$ Therefore, for different localized lasing modes, even with significant spatial or frequency overlap, the interactions between modes must be frozen above the RL threshold. Notice that photons from different localized lasing modes would be strongly uncorrelated (for a representative picture, see Figure S7). We note that the similarity in intensity observed in the spectrum of the small micrometric peaks $(\sim 7-8 \mathrm{~nm}$; Figure $3 \mathrm{f})$ is broader than that observed in the spectrum of the peaks mode extracted through the integrated collection over the whole emission volume $(\sim 2-$ $3 \mathrm{~nm}$, Figure 3d). This must be because the acquisition depth for the small micrometric collection $(<4 \mu \mathrm{m})$ is shallower than that for the integrated collection $(\sim 15 \mu \mathrm{m})$. In this way, the peaks mode spectrum, collected by integrating over the whole emission volume, represents modes with a range of quality factors broader than that from the micrometric collection (near the input-pumping surface). The quality factor of a passive mode at localization must decrease with depth, as localization decreases with depth. $5,48,49$

Figure $3 \mathrm{~g}$ shows the behavior of the spectrally integrated RL emission for the frontal integrated collection (collected over the whole emission volume) and back collection (in exact analogy to Figure 3a where we analyzed the peak spectral intensity instead). Figure $3 \mathrm{~h}$ shows a comparison of the spectrally integrated RL emissions for peaks and band modes. The integrated emission slope (fluencies $\leq 8.4 \mathrm{~mJ} \mathrm{~cm}^{-2}$ ) of the peaks mode (green line) was $\sim 2.9$ times higher than that for the extended mode band (magenta line). Therefore, for fluencies $\leq 8.4 \mathrm{~mJ} \mathrm{~cm}^{-2}, \sim 74 \%$ of the $\mathrm{RL}$ emission, detected with our experimental setup, corresponds to the peaks mode. 
A detailed study with a detection system of more resolution and sensibility is called for, to more precisely determine the linewidth of the RL peaks and their relationship with $\tau_{\mathrm{eO}}$, as well as the physical origin of $\tau_{\mathrm{eO}}$. Moreover, an expanded study varying the pumping and collection angles ${ }^{50}$ would allow a better understanding of the $\mathrm{RL}$ operation at the localization transition.

\section{CONCLUSIONS}

Core-shell TiO $\mathrm{TiO}_{2} @$ Silica NPs at $\left[140 \times 10^{10} \mathrm{NPs} \mathrm{mL}^{-1}\right]$ in an ethanol solution of R6G $\left[1 \times 10^{-4} \mathrm{M}\right]$ allowed us to study the $\mathrm{RL}$ action at the localization transition. An absorptionemission saturation phenomenon was observed in the RL. This phenomenon was associated with the absorptionemission saturation of the localized modes called the peaks mode. A large redshift of $\sim 7.6 \mathrm{~nm}$ is observed in the $\mathrm{RL}$ emission spectrum, which was associated with the emission of the peaks mode. The FAP measurement provides an additional approach to the study of the scattering medium, which allows us to infer that the localized modes must be confined near the input-pumping border $\left(\mathrm{FAP}_{(\mu \mathrm{m})}\right)$. In addition, $\operatorname{FAP}_{(\mu \mathrm{m})}$ measurement allowed us to infer that the absorption above the RL threshold does not suppress localizing pumping photons. The latter indicates that the frequency and wavefunction of a localized lasing mode should be approximately the same as those of the passive mode of the scattering medium. Narrow peaks with a linewidth of $\leq 0.17 \mathrm{~nm}$ were observed in the $\mathrm{RL}$ emission spectrum. This linewidth $(0.17 \mathrm{~nm})$ is narrower than the mean linewidth of the modes of the scattering medium $\left(\sim 1.29 \mathrm{~nm}, \tau_{\mathrm{eO}(\text { Micro })} \approx 0.612 \mathrm{ps}\right)$. This fact could be a consequence of the anomalous nonlinear increase of the refractive index by localization, due to the continuously increasing intensity within the closed loop paths during the residence time of the pumping photons. This effect would be greatly suppressed for emitted photons above the lasing threshold, as the intensity increase would be a result of stimulated emission. The back-collection method allowed for the separate measurement of the RL band mode, showing a linear dependence with pumping fluence without gain depletion. The emission spectrum of the peaks mode was extracted by subtracting the frontal and back spectra, showing narrow peaks with similar intensity in a broad frequency range. The latter suggests suppression of interaction between the peaks modes, which is a signature of random lasing at localization. Finally, the contribution of RL peaks and band modes was determined.

\section{MATERIALS AND METHODS}

The pumping source of the RL was the second harmonic of a Q-switched Nd:YAG laser system with a pulse width of $\sim 4 \mathrm{~ns}$, a repetition rate up to $15 \mathrm{~Hz}$, and a spot size of $3 \mathrm{~mm}$. The emission spectra were collected through a multimode optical fiber (50 or $200 \mu \mathrm{m})$, coupled to a spectrometer HR4000 UVVIS (Ocean Optics), with a $0.17 \mathrm{~nm}$ spectral resolution (FWHM). The pumping energy fluence per pulse is varied between 0.003 and $72 \mathrm{~mJ} \mathrm{~cm}{ }^{-2}$. The schematic diagrams of the experimental setup for $\mathrm{RL}$ studies are shown in Figures $\mathrm{S} 1 \mathrm{a}, \mathrm{b}$ and S5a. To study in depth the emission spectrum (emerging peaks), RL emission coming from a micrometric region near the input-pumping surface was collected through an appropriate optical system (Figure S1b), using the same pumping spot ( $3 \mathrm{~mm}$ diameter). The emission spectra were acquired by integrating 21 laser shots $(1.4 \mathrm{~s}$ at $15 \mathrm{~Hz})$. R6G at $\left[1 \times 10^{-4}\right.$ $\mathrm{M}$ ] was added to the ethanol solution containing $\mathrm{TiO}_{2} @$ Silica $\mathrm{NPs}$ at $\left[140 \times 10^{10} \mathrm{NPs} \mathrm{mL}^{-1}\right]$. The transport mean free path $\left(l_{\mathrm{T}}\right)$ for this [NPs] was determined through the measurement of the transmitted coherent intensity $\left(l_{\mathrm{T} 0}=1.3 \mu \mathrm{m}\right){ }^{5}$

\section{ASSOCIATED CONTENT}

\section{Supporting Information}

The Supporting Information is available free of charge on the ACS Publications website at DOI: 10.1021/acsomega.7b00086.

Description of the $\mathrm{TiO}_{2} @$ Silica NPs synthesis; experimental setup for RL study; frontal emission spectra of the RL; potential curves of the $S_{0 i}$ and $S_{1 i}$ electronic states (R6G); emission spectra collected from a micrometric volume near the input interface; experimental setup for back collection and $\mathrm{RL}$ emission spectra collected by this back-collection method; comparison between frontal and back spectra (integrated collection); representative picture of suppression of the interaction between localized modes (PDF)

\section{AUTHOR INFORMATION}

\section{Corresponding Author}

*E-mail: Ernesto.Jimenez@uv.es. ORCID $\odot$

Ernesto Jiménez-Villar: 0000-0002-9049-5881

Paulo C. de Oliveira: 0000-0001-9607-5186

\section{Author Contributions}

All authors conceived and designed the experiments; E.J.-V., I.F.S., and V.M. performed the experiments; E.J.-V., N.U.W., and C.L. analyzed the results. E.J.-V. guided the research and wrote the manuscript.

\section{Notes}

The authors declare no competing financial interest.

\section{ACKNOWLEDGMENTS}

We gratefully acknowledge financial support from FACEPE, FAPESP, FAPESQ and CNPq (Brazil). E.J.-V. acknowledges FACEPE (grant 0116-1.06/13, APQ-0071-1.06/14) and FAPESQ. I.F.S. thanks the CAPES (Brazil) for the fellowships of masters. N.U.W. acknowledges CNPq (grants 402241 2012_6 and 307989 2013-5) and FAPESP (grant 18162-4 2012). C.L. acknowledges Spanish MINECO (grant MAT2015-68075-R (SIFE)) and the Comunidad de Madrid (grant S2013/MIT-2740 (PHAMA_2.0)). We thank engineers Ricardo Acosta and Colin Roxburgh, for their corrections to the text. We extend additional thanks to designer Pedro Silva for the graphical image.

\section{REFERENCES}

(1) John, S. Electromagnetic absorption in a disordered medium near a photon mobility edge. Phys. Rev. Lett. 1984, 53, No. 2169.

(2) Anderson, P. W. The question of classical localization A theory of white paint? Philos. Mag. B 1985, 52, 505-509.

(3) Wiersma, D. S.; Bartolini, P.; Lagendijk, A.; Righini, R. Localization of light in a disordered médium. Nature 1997, 390, 671-673.

(4) Sperling, T.; Buhrer, W.; Aegerter, C. M.; Maret, G. Direct determination of the transition to localization of light in three dimensions. Nat. Photonics 2013, 7, 48-52.

(5) Jimenez-Villar, E.; et al. Anderson localization of light in a colloidal suspension ( $\mathrm{TiO}_{2} @$ Silica). Nanoscale 2016, 8, 10938-10946. 
(6) Bromberg, Y.; Lahini, Y.; Small, E.; Silberberg, Y. Hanbury Brown and Twiss interferometry with interacting photons. Nat. Photonics 2010, 4, 721-726.

(7) Firstenberg, O.; et al. Attractive photons in a quantum nonlinear medium. Nature 2013, 502, 71-75.

(8) Wang, H.; et al. Single-crystalline rutile $\mathrm{TiO}_{2}$ hollow spheres: room-temperature synthesis, tailored visible-light-extinction, and effective scattering layer for quantum dot-sensitized solar cells. J. Am. Chem. Soc. 2011, 133, 19102-19109.

(9) Cernuto, G.; et al. Size and shape dependence of the photocatalytic activity of $\mathrm{TiO}_{2}$ nanocrystals: a total scattering debye function study. J. Am. Chem. Soc. 2011, 133, 3114-3119.

(10) Leonetti, M.; Conti, C.; Lopez, C. The mode-locking transition of random lasers. Nat. Photonics 2011, 5, 615-617.

(11) Stano, R.; Jacquod, P. Suppression of interactions in multimode random lasers in the Anderson localized regime. Nat. Photonics 2013, $7,66-71$.

(12) Liu, J.; et al. Random nanolasing in the Anderson localized regime. Nat. Nanotechnol. 2014, 9, 285-289.

(13) Demirörs, A. F.; Blaaderen, A. V.; Imhof, A. Synthesis of eccentric titania-silica core-shell and composite particles. Chem. Mater. 2009, 21, 979-984.

(14) Abderrafi, K.; et al. Production of nanometer-size GaAs nanocrystals by nanosecond laser ablation in liquid. J. Nanosci. Nanotechnol. 2012, 12, 6774-6778.

(15) Jimenez-Villar, E.; Mestre, V.; Oliveira, P. C.; de Sá, G. F. Novel core-shell $\left(\mathrm{TiO}_{2} @\right.$ Silica $)$ nanoparticles for scattering medium in a random laser: higher efficiency, lower laser threshold and lower photodegradation. Nanoscale 2013, 5, 12512-12517.

(16) Skipetrov, S. E.; Sokolov, I. M. Absence of Anderson localization of light in a random ensemble of point scatterers. Phys. Rev. Lett. 2014, 112, No. 023905.

(17) Jimenez-Villar, E.; et al. $\mathrm{TiO}_{2} @$ Silica nanoparticles in a random laser: Strong relationship of silica shell thickness on scattering medium properties and random laser performance. Appl. Phys. Lett. 2014, 104, No. 081909.

(18) Jiménez, E.; et al. Laser-ablation-induced synthesis of $\mathrm{SiO}_{2-}$ capped noble metal nanoparticles in a single step. Langmuir 2010, 26, $7458-7463$.

(19) González-Castillo, J. R.; et al. Synthesis of Ag@Silica Nanoparticles by Assisted Laser Ablation. Nanoscale Res. Lett. 2015, 10, No. 399.

(20) Ermakov, V. A.; et al. Size Control of Silver-Core/Silica-Shell Nanoparticles Fabricated by Laser-Ablation-Assisted Chemical Reduction. Langmuir 2017, 33, 2257-2262.

(21) Rodriquez, E.; et al. All-optical switching device for infrared based on PbTe quantum dots. Superlattices Microstruct. 2008, 43, 626634.

(22) Rodríguez, E.; et al. $\mathrm{SiO}_{2} / \mathrm{PbTe}$ quantum-dot multilayer production and characterization. Appl. Phys. Lett. 2005, 86, No. 113117.

(23) Fuertes, G.; et al. Switchable bactericidal effects from novel silica-coated silver nanoparticles mediated by light irradiation. Langmuir 2011, 27, 2826-2833.

(24) Kellermann, G.; et al. Structure of $\mathrm{PbTe}\left(\mathrm{SiO}_{2}\right) / \mathrm{SiO}_{2}$ multilayers deposited on $\mathrm{Si}(111)$. J. Appl. Crystallogr. 2010, 43, 385-393.

(25) Rodriguez, E.; et al. Fabrication and characterization of a $\mathrm{PbTe}$ quantum dots multilayer structure. Phys. E 2005, 26, No. 361.

(26) Pradhan, P.; Kumar, N. Localization of light in coherently amplifying random media. Phys. Rev. B: Condens. Matter Mater. Phys. 1994, 50, 9644-9647.

(27) Cao, H.; et al. Spatial confinement of laser light in active random media. Phys. Rev. Lett. 2000, 84, 5584.

(28) Wiersma, D. S. The physics and applications of random lasers. Nat. Phys. 2008, 4, 359-367.

(29) Noginov, M. A.; et al. Demonstration of a spaser-based nanolaser. Nature 2009, 460, 1110-1113.

(30) Cao, H.; et al. Random laser action in semiconductor powder. Phys. Rev. Lett. 1999, 82, 2278.
(31) $\mathrm{Wu}, \mathrm{X}$; ; et al. Random lasing in closely packed resonant scatterers. J. Opt. Soc. Am. B 2004, 21, 159-167.

(32) van der Molen, K. L.; Tjerkstra, R. W.; Mosk, A. P.; Lagendijk, A. Spatial extent of random laser modes. Phys. Rev. Lett. 2007, 98, No. 143901.

(33) Leonetti, M.; lopez, C. Random lasing in structures with multiscale transport properties. Appl. Phys. Lett. 2012, 101, No. 251120.

(34) Conti, C.; Fratalocchi, A. Dynamic light diffusion, threedimensional Anderson localization and lasing in inverted opals. Nat. Phys. 2008, 4, 794-798.

(35) Polson, R. C.; Chipouline, A.; Vardeny, Z. V. Random lasing in $\pi$-conjugated films and infiltrated opals. Adv. Mater. 2001, 13, 760764

(36) Gagné, M.; Kashyap, R. Demonstration of a $3 \mathrm{~mW}$ threshold Erdoped random laser base don a unique fiber brating. Opt. Express 2009, 17, 19067-19074.

(37) Turitsyn, S. K. et al. Random distributed feedback fibre laser. Nat. Photonics 2010, 4, 231-235.

(38) Mujumdar, S.; Ricci, M.; Torre, R.; Wiersma, D. S. Amplified extended modes in random lasers. Phys. Rev. Lett. 2004, 93, No. 053903.

(39) Wu, X.; Andreasen, J.; Cao, H.; Yamilov, A. Effect of local pumping on random laser modes in one dimension. J. Opt. Soc. Am. B 2007, 24, A26.

(40) Wu, X.; Cao, H. Statistical studies of random-lasing modes and amplified spontaneous-emission spikes in weakly scattering systems. Phys. Rev. A 2008, 77, No. 013832.

(41) Türeci, H. E.; Ge, L.; Rotter, S.; Stone, A. D. Strong interactions in multimode random lasers. Science 2008, 320, 643-646.

(42) Zaitsev, O.; Deych, L. Recent developments in the theory of multimode random lasers. J. Opt. 2010, 12, No. 024001.

(43) Noginov, M. A.; Caulfield, H. J.; Noginova, N. E.; Venkateswarlu, P. Line narrowing in the dye solution with scattering centers. Opt. Commun. 1995, 118, 430-437.

(44) John, S.; Wang, J. Quantum electrodynamics near a photonic band gap: Photon bound states and dressed atoms. Phys. Rev. Lett. 1990, 64, 2418.

(45) John, S. Localization of light. Phys. Today 1991, 44, 32.

(46) Yablonovitch, E. Inhibited Spontaneous Emission in Solid-State Physics and Electronics. Phys. Rev. Lett. 1987, 58, 2059.

(47) Buttiker, M.; Moskalets, M. From Anderson Localization to Mesoscopic Physics. Int. J. Mod. Phys. B 2010, 24, 1555-1576.

(48) Mirlin, A. D. Spatial structure of anomalously localized states in disordered conductors. J. Math. Phys. 1997, 38, 1888.

(49) Mirlin, A. D. Statistics of energy levels and eigenfunctions in disordered systems. Phys. Rep. 2000, 326, 259-382.

(50) Jimenez-Villar, E.; et al. Anderson localization of light: Strong dependence with incident angle. arXiv:1705.09262 and arXiv:1705.09645. 